\title{
A cooperative game-theoretic approach to ALOHA
}

Citation for published version (APA):

Marban, S., Ven, P., Borm, P. J. A., \& Hamers, H. (2010). A cooperative game-theoretic approach to ALOHA. METEOR, Maastricht University School of Business and Economics. METEOR Research Memorandum No. 049 https://doi.org/10.26481/umamet.2010049

Document status and date:

Published: 01/01/2010

DOI:

10.26481/umamet.2010049

Document Version:

Publisher's PDF, also known as Version of record

\section{Please check the document version of this publication:}

- A submitted manuscript is the version of the article upon submission and before peer-review. There can be important differences between the submitted version and the official published version of record.

People interested in the research are advised to contact the author for the final version of the publication, or visit the DOI to the publisher's website.

- The final author version and the galley proof are versions of the publication after peer review.

- The final published version features the final layout of the paper including the volume, issue and page numbers.

Link to publication

\footnotetext{
General rights rights.

- You may freely distribute the URL identifying the publication in the public portal. please follow below link for the End User Agreement:

www.umlib.nl/taverne-license

Take down policy

If you believe that this document breaches copyright please contact us at:

repository@maastrichtuniversity.nl

providing details and we will investigate your claim.
}

Copyright and moral rights for the publications made accessible in the public portal are retained by the authors and/or other copyright owners and it is a condition of accessing publications that users recognise and abide by the legal requirements associated with these

- Users may download and print one copy of any publication from the public portal for the purpose of private study or research.

- You may not further distribute the material or use it for any profit-making activity or commercial gain

If the publication is distributed under the terms of Article $25 \mathrm{fa}$ of the Dutch Copyright Act, indicated by the "Taverne" license above, 


\section{Maastricht University}

Sebastian Marban, Peter van de Ven, Peter Borm, Herbert Hamers

A cooperative game-theoretic approach to ALOHA

$\mathrm{RM} / 10 / 049$

\section{METEOR}

Maastricht University School of Business and Economics

Maastricht Research Schocl of Economics

of Technology and Organization

PO. Box 616

NL - 6200 MD Mastricht

The Netherlands 


\title{
A cooperative game-theoretic approach to ALOHA
}

\author{
Sebastian Marban ${ }^{1} \quad$ Peter van de Ven $^{2,3} \quad$ Peter Borm ${ }^{4}$ \\ Herbert Hamers ${ }^{4}$ \\ ${ }^{1}$ Department of Quantitative Economics \\ Maastricht University \\ P.O. Box 616, 6200 MD Maastricht, The Netherlands \\ ${ }^{2}$ Department of Mathematics \& Computer Science \\ Eindhoven University of Technology \\ P.O. Box 513, 5600 MB Eindhoven, The Netherlands \\ ${ }^{3}$ EURANDOM, P.O. Box 513, 5600 MB Eindhoven, The Netherlands \\ ${ }^{4}$ Department of Econometrics and Operations Research \\ Tilburg University \\ P.O. Box 90153, 5000 LE Tilburg, The Netherlands
}

\begin{abstract}
The performance of wireless networks suffers from collisions. These occur when multiple wireless nodes transmit simultaneously, and their signals interfere with each other. To reduce collisions, nodes may use a randomized protocol to regulate their behavior. An example of such a protocol is slotted ALOHA, in which at the beginning of each time slot all nodes have a fixed probability to start a transmission for the duration of that slot. In this paper we consider a wireless network where all nodes use slotted ALOHA, and we investigate the impact of cooperation among nodes. Full cooperation eliminates collisions between nodes, which clearly has a positive impact on the network performance, measured by the aggregate node throughput.

We are interested in how to allocate the corresponding performance gains obtained from cooperation among the nodes. In order to study this allocation problem, we define and analyze a corresponding cooperative ALOHA game. We show that this type of game is convex and we consider three solution concepts: the core, the Shapley value, and the compromise value. Further, we introduce the set of Weighted Gain Splitting (WGS) allocation rules, and show that this set coincides with the core of the game. These WGS rules can be used to provide an alternative characterization of the Shapley value. Finally, we analyze how the cooperative solution concepts change with the parameters of the wireless network.
\end{abstract}

Keywords: ALOHA protocol, cooperative games, compromise value, core, Shapley value, wireless networks

\section{Introduction}

Wireless networks have gone through enormous changes in the recent past, developing from academic curiosities to a part of every-day life in the span of a few decades. A key characteristic of wireless networks is that nodes share the same medium (air) for their transmissions. When two 
or more nodes transmit simultaneously, the intended receiver receives multiple signals, and may be unable to distinguish between them. When such a collision occurs, none of the transmissions will be received correctly.

When designing a wireless network, it is important to regulate transmissions, as collisions have a strong negative impact on the network performance. Transmissions may be regulated in two ways: (i) through a centralized controller that schedules the transmissions of all nodes; or (ii) by using a distributed mechanism, so that each node for itself decides when to transmit. An example of the former is GSM, where a base station regulates the activity of the cell phones, while the latter can be illustrated by for example wireless LAN. In this paper we study the distributed scenario.

For distributed networks, a so-called Medium Access Control (MAC) protocol is used to control transmissions. The earliest example of such a MAC protocol is (pure) ALOHA, see Abramson [1]. Nodes using this protocol start a transmission whenever they have a packet available in their buffer. If the transmission is finished and no acknowledgment is received signaling that the transmission was successful (contrary to wired communications, unsuccessful wireless transmissions cannot be detected), the node waits for a random time before retransmitting. This mechanism introduces randomness into the system, and prevents repeated collisions.

The ALOHA protocol was subsequently refined to slotted ALOHA by Roberts [10]. This protocol introduces a number of changes compared to pure ALOHA, the most important of which is the partition of time into slots. At the beginning of a time slot, each node for itself decides whether to transmit for the duration of a single slot. By synchronizing all nodes and uniformizing the transmission duration, slotted ALOHA eliminates collisions that occur halfway through a transmission. Instead, collisions can occur only at the beginning of each slot. Roberts shows that this revised protocol significantly outperforms pure ALOHA. Although many other protocols have been introduced since ALOHA, is still being used. In particular for applications that require a simple protocol, and do not demand high performance. Examples include the transmission of control messages in satellite ground stations [11] and cellular networks.

In this paper we consider a wireless network where the activity of nodes is governed by slotted ALOHA. The network performance is measured by the normalized throughput, that is, the fraction of slots in which a successful transmission takes place. Generalizing the typical behavior of such networks, we allow nodes to cooperate by adapting their transmissions to each other such that no collisions occur, even if multiple nodes transmit simultaneously. However, the cooperation is such that, even when multiple nodes transmit in the same slot, only one successful transmission can be achieved. Cooperation will increase the network performance significantly, as more slots will be occupied by a successful transmission. We want to know how to allocate these joint throughput gains among the nodes.

To analyze this allocation problem, we consider a corresponding cooperative game, called an ALOHA game. We show that this type of game is convex and we obtain an explicit expression for the compromise value. Further, we introduce a class of rules, so called Weighted Gain Splitting (WGS) rules, that allocate the gains without explicit knowledge of the game. For each wireless network situation, this class of rules turns out to coincide with the core of the game. The WGS rules also allow us to give an alternative characterization of the Shapley value. Using this characterization, we investigate how the Shapley value responds to changes in the network.

Cooperative game theory has been applied to study wireless networks occasionally. For example, Mathur et al. [9] discuss a Gaussian interference channel where nodes are allowed to cooperate by jointly decoding the received signals. La and Anantharam [8] consider a setting where nodes might jam the channel for other users, coercing other nodes to give up a larger share of the channel. Finally, Aram et al. [2] consider a setting where wireless service providers may share base stations and spectrum, and discuss how to design this cooperation.

The remainder of this paper is structured as follows. Section 2 discusses some background of cooperative game theory. In Section 3, we present a wireless network using ALOHA, and we outline the nature of the cooperation. We introduce a set of division rules that allocate the gains from cooperation among the nodes. Section 4 introduces and analyses the corresponding cooperative game. We consider three solution concepts in Section 5: the core, the Shapley value, and the compromise value. In Section 6 we study how the allocations resulting from the proposed 
solution concepts change with the parameters of the wireless network.

\section{Preliminaries on cooperative games}

A cooperative game with transferable utility (TU-game) is characterized by a pair $(N, v)$ consisting of a non-empty, finite set of players $N$ and the characteristic function $v: 2^{N} \rightarrow \mathbb{R}$ with $v(\emptyset)=0$. Subsets of $N$ are called coalitions, and $v(S)$ represents the value of coalition $S \subseteq N$. An important property of TU-games is convexity. A game $(N, v)$ is called convex if for all $i \in N$ and $S \subseteq T \subseteq$ $N \backslash\{i\}$,

$$
v(S \cup\{i\})-v(S) \leq v(T \cup\{i\})-v(T) .
$$

Thus, for a convex game, the marginal contribution of a player joining a coalition is greater than the marginal contribution for joining any subset of this coalition.

Assume that all players negotiate the formation of the grand coalition $N$. We are interested in how to divide the value $v(N)$ among the players, taking into account the values $v(S)$ of all possible coalitions $S \subseteq N$. Let $x_{i}$ represent the payoff to player $i$ and let $x \in \mathbb{R}^{N}$ with $\sum_{i \in N} x_{i}=v(N)$ denote a payoff vector. A solution concept assigns a payoff vector or a set of payoff vectors to a game. In this paper we are concerned with three solution concepts: the core introduced by Gillies [5], the Shapley value introduced by Shapley [12], and the compromise value introduced by Tijs [14].

The core of a game is defined as

$$
C(v)=\left\{x \in \mathbb{R}^{N} \mid \sum_{i \in N} x_{i}=v(N), \forall_{S \subseteq N} \sum_{i \in S} x_{i} \geq v(S)\right\} .
$$

In general, the core of a game may be empty. Shapley [13], however, proved that convex games have non-empty cores.

Let $\Pi(N)$ denote the set of all permutations of $N$ and let $\sigma \in \Pi(N)$, where $\sigma(i)=k$ means that player $i$ is at the $k$-th position. We define the marginal vector $m^{\sigma}(v) \in \mathbb{R}^{N}$ with respect to $\sigma \in \Pi(N)$ as

$$
m_{i}^{\sigma}(v)=v\left(\Omega^{\sigma}(\sigma(i))\right)-v\left(\Omega^{\sigma}(\sigma(i)-1)\right), \quad i \in N,
$$

with $\Omega^{\sigma}(k)=\{j \in N \mid \sigma(j) \leq k\}$ and $m_{i}^{\sigma}$ the $i$-th component of $m^{\sigma}$. Shapley [13] and Ichiishi [7] showed that the core of a convex game may be written as the convex hull of all marginal vectors

$$
C(v)=\operatorname{conv}\left\{m^{\sigma}(v) \mid \sigma \in \Pi(N)\right\} .
$$

The Shapley value $\Phi(v)$ of TU-game $(N, v)$ is defined as the average of all marginal vectors, i.e.,

$$
\Phi(v)=\frac{1}{|N| !} \sum_{\sigma \in \Pi(N)} m^{\sigma}(v)
$$

Finally, the compromise value $\tau(v)$ of convex game is determined by

$$
\tau(v)=\beta M(v)+(1-\beta) m(v),
$$

where $\beta$ is the unique element of $[0,1]$ such that $\sum_{i \in N} \tau_{i}(v)=v(N)$,

$$
M_{i}(v)=v(N)-v(N \backslash\{i\}) \quad \text { and } \quad m_{i}(v)=v(\{i\}), \quad i \in N .
$$




\section{ALOHA situations}

Consider a wireless network consisting of a set of nodes $N=\{1, \ldots, n\}$. Time is slotted, and at the beginning of each slot, node $i \in N$ transmits a packet with probability $p_{i}, 0<p_{i} \leq 1$, independent of the other nodes. Transmissions occupy a single slot, and may be successful or not depending on the number of nodes transmitting simultaneously and on the node interaction. We are interested in the throughput $\theta_{i}$ of node $i$, defined as the fraction of slots in which node $i$ is engaged in a successful transmission. In the remainder, we assume that this throughput can be expressed in an infinitely divisible transferable commodity.

An $A L O H A$ situation as described above is characterized by the pair $(N, p)$, with $N$ the set of players, and $p=\left(p_{1}, p_{2}, \ldots, p_{n}\right)$ the vector of all transmission probabilities. We compare two types of interaction between nodes: with cooperation and without cooperation. In the setting without cooperation, node $i$ performs a successful transmission if and only if it is the only node to transmit in a particular time slot. So,

$$
\theta_{i}=p_{i} \prod_{j \in N \backslash\{i\}}\left(1-p_{j}\right), \quad i \in N .
$$

The aggregate throughput $\theta$ is obtained by summing over all nodes $i \in N$,

$$
\theta=\sum_{i \in N} p_{i} \prod_{j \in N \backslash\{i\}}\left(1-p_{j}\right) .
$$

Note that $0 \leq \theta \leq 1$, as in each slot at most one successful transmission takes place.

In the setting with cooperation, transmissions are coordinated in such a way that even when multiple nodes transmit in the same time slot no collisions occur. However, in each time slot at most a single successful transmission can take place. Under these assumptions, the aggregate throughput $\theta(N)$ is the probability that at least one node transmits, i.e.,

$$
\theta(N)=1-\prod_{i \in N}\left(1-p_{i}\right)
$$

Combining (6) and (7) we obtain the throughput gain from cooperation,

$$
\theta(N)-\theta=1-\prod_{i \in N}\left(1-p_{i}\right)-\sum_{i \in N} p_{i} \prod_{j \in N \backslash\{i\}}\left(1-p_{j}\right) .
$$

To illustrate an ALOHA situation, we discuss the following example.

Example 1. Consider the ALOHA situation $(N, p)$ with $N=\{1,2,3\}$ and $p=(0.4,0.3,0.5)$. By (5) and (6) we then have

$$
\begin{aligned}
\theta_{1} & =0.4 \cdot(1-0.3)(1-0.5)=0.14, \\
\theta_{2} & =0.3 \cdot(1-0.4)(1-0.5)=0.09, \\
\theta_{3} & =0.5 \cdot(1-0.4)(1-0.3)=0.21, \\
\theta & =\sum_{i \in N} \theta_{i}=0.14+0.09+0.21=0.44 .
\end{aligned}
$$

Using (7) one finds that

$$
\theta(N)=1-(1-0.4)(1-0.3)(1-0.5)=0.79 .
$$

Hence, by (8) the throughput gain from cooperation is

$$
\theta(N)-\theta=0.79-0.44=0.35 .
$$


We derive the following alternative expression for (8).

Proposition 1. Let $(N, p)$ be an ALOHA situation. The throughput gain induced by cooperation equals

$$
\theta(N)-\theta=\sum_{\substack{D \subseteq N \\|D| \geq 2}} \prod_{i \in D} p_{i} \prod_{i \in N \backslash D}\left(1-p_{i}\right)
$$

Proof. This readily follows from (8) and the identity

$$
\sum_{k=0}^{n} \sum_{\substack{D \subseteq N \\|D|=k}} \prod_{i \in D} p_{i} \prod_{i \in N \backslash D}\left(1-p_{i}\right)=1 .
$$

We conclude from Proposition 1 that the aggregate node throughput increases under cooperation, and that node cooperation is profitable when at least two nodes are transmitting. We are interested in how to divide these gains. A set of division rules that take into account the contribution of different nodes to the gain is the set of Weighted Gain Splitting (WGS) rules, that allocates the throughput gains from cooperation according to node activity. Specifically, given a set $D \subseteq N$ of transmitting nodes. Node $i \in D$ receives a fraction $\lambda_{i}^{D}$ of the gains induced by cooperation. Let $\Lambda$ be the set of all such coalitional fraction vectors, i.e.,

$$
\Lambda=\left\{\lambda=\left\{\lambda^{D}\right\}_{D \subseteq N} \subseteq \mathbb{R}^{N} \mid \forall_{D \subseteq N} \forall_{i \in D} \lambda_{i}^{D} \geq 0, \forall_{D \subseteq N} \forall_{i \in N \backslash D} \lambda_{i}^{D}=0, \forall_{D \subseteq N} \sum_{i \in D} \lambda_{i}^{D}=1\right\} .
$$

For any $\lambda \in \Lambda$, the corresponding weighted gain splitting rule $W G S^{\lambda}$ allocates the gains from cooperation in an ALOHA situation $(N, p)$ according to

$$
W G S_{i}^{\lambda}(p)=\sum_{\substack{D \subseteq N \\ i \in D \\|D| \geq 2}} \lambda_{i}^{D} \prod_{j \in D} p_{j} \prod_{j \in N \backslash D}\left(1-p_{j}\right), \quad i \in N .
$$

So each WGS rule allocates a non-negative value to each node. The set of all possible WGS allocations is given by

$$
W G S(p)=\left\{W G S^{\lambda}(p) \mid \lambda \in \Lambda\right\} .
$$

It is readily seen that $W G S(p)$ is convex.

We show that the WGS rules are efficient in the sense that they allocate all throughput gain.

Proposition 2. Let $(N, p)$ be an $A L O H A$ situation and let $\lambda \in \Lambda$. Then,

$$
\sum_{i \in N} W G S_{i}^{\lambda}(p)=\theta(N)-\theta .
$$

Proof. From (10), we get

$$
\begin{aligned}
\sum_{i \in N} W G S_{i}^{\lambda}(p) & =\sum_{i \in N} \sum_{\substack{D \subseteq N \\
i \in D \\
|D| \geq 2}} \lambda_{i}^{D} \prod_{j \in D} p_{j} \prod_{j \in N \backslash D}\left(1-p_{j}\right) \\
& =\sum_{\substack{D \subseteq N \\
|D| \geq 2}} \sum_{i \in D} \lambda_{i}^{D} \prod_{j \in D} p_{j} \prod_{j \in N \backslash D}\left(1-p_{j}\right) \\
& =\sum_{\substack{D \subseteq N \\
|D| \geq 2}} \prod_{j \in D} p_{j} \prod_{j \in N \backslash D}\left(1-p_{j}\right)=\theta(N)-\theta,
\end{aligned}
$$

where the last equality follows from Proposition 1. 


\section{ALOHA games}

To analyze in more detail the possible allocations, we turn to cooperative game theory and incorporate coalitional considerations. To this end, we introduce a cooperative game corresponding to each ALOHA situation.

Consider a coalition $S \subseteq N$ of cooperating nodes. Simultaneous transmissions by nodes in $S$ do not interfere with each other. So a transmission from $S$ is successful if and only if all nodes in $N \backslash S$ remain silent, and the total throughput $\theta(S)$ of the coalition $S$ is given by

$$
\theta(S)=\left(1-\prod_{i \in S}\left(1-p_{i}\right)\right) \prod_{i \in N \backslash S}\left(1-p_{i}\right) .
$$

Every ALOHA situation $(N, p)$ gives rise to an ALOHA game $(N, v)$, where the reward of coalition $S$ is defined as the difference between the throughput of the nodes in $S$ with cooperation and the throughput without cooperation,

$$
v(S)=\theta(S)-\sum_{i \in S} \theta_{i}
$$

Analogous to Proposition 1, we find the following alternative expression for the coalitional gains.

Proposition 3. Let $(N, v)$ be an ALOHA game corresponding to an ALOHA situation $(N, p)$. Then,

$$
v(S)=\sum_{\substack{D \subseteq S \\|D| \geq 2}} \prod_{i \in D} p_{i} \prod_{i \in N \backslash D}\left(1-p_{i}\right), \quad S \subseteq N .
$$

Proof. Let $S \subseteq N$. By (11) and (12), we get

$$
\begin{aligned}
v(S) & =\left(1-\prod_{i \in S}\left(1-p_{i}\right)\right) \prod_{i \in N \backslash S}\left(1-p_{i}\right)-\sum_{i \in S} p_{i} \prod_{j \in N \backslash\{i\}}\left(1-p_{j}\right) \\
& =\prod_{i \in N \backslash S}\left(1-p_{i}\right)\left(1-\prod_{i \in S}\left(1-p_{i}\right)-\sum_{i \in S} p_{i} \prod_{j \in S \backslash\{i\}}\left(1-p_{j}\right)\right) \\
& =\prod_{i \in N \backslash S}\left(1-p_{i}\right)\left(\sum_{\substack{D \subseteq S \\
|D| \geq 2}} \prod_{i \in D} p_{i} \prod_{i \in S \backslash D}\left(1-p_{i}\right)\right) \\
& =\sum_{\substack{D \subseteq S \\
|D| \geq 2}} \prod_{i \in D} p_{i} \prod_{i \in N \backslash D}\left(1-p_{i}\right) .
\end{aligned}
$$

Note that for $S=N$, the coalitional gain (13) reduces to the total gain found in (9). When $S$ consists of a single node we see that $v(S)=0$.

To illustrate the ALOHA game $(N, v)$, we revisit Example 1.

Example 2. By (11), we can determine $\theta(S)$ for all possible coalitions $S$, i.e.,

$$
\begin{aligned}
& \theta(1)=\theta_{1}=0.14, \quad \theta(2)=\theta_{2}=0.09, \quad \theta(3)=\theta_{3}=0.21, \\
& \theta(\{1,2\})=(1-0.5)[1-(1-0.4)(1-0.3)]=0.29 \\
& \theta(\{2,3\})=(1-0.4)[1-(1-0.3)(1-0.5)]=0.39 \\
& \theta(\{1,3\})=(1-0.3)[1-(1-0.4)(1-0.5)]=0.49 \\
& \theta(\{1,2,3\})=1-(1-0.4)(1-0.3)(1-0.5)=0.79 .
\end{aligned}
$$

The ALOHA game $(N, v)$ corresponding to the ALOHA situation of Example 1 is therefore determined by 


\begin{tabular}{|c||c|c|c|c|c|c|c|}
\hline$S$ & 1 & 2 & 3 & 12 & 23 & 13 & 123 \\
\hline$v(S)$ & 0 & 0 & 0 & 0.06 & 0.09 & 0.14 & 0.35 \\
\hline
\end{tabular}

Consider for example, $v(\{2,3\})=0.09$, which can be determined either by subtracting $0.09\left(\theta_{2}\right)$ and $0.21\left(\theta_{3}\right)$ from $0.39(\theta(\{2,3\}))$ or directly by (13):

$$
\begin{aligned}
v(\{2,3\}) & =\prod_{i \in\{2,3\}} p_{i} \prod_{i \in\{1\}}\left(1-p_{i}\right) \\
& =0.3 \cdot 0.5(1-0.4)=0.09 .
\end{aligned}
$$

In showing that any ALOHA game is convex, we use the following auxiliary lemma.

Lemma 1. Let $(N, p)$ be an ALOHA situation and let $(N, v)$ be the corresponding ALOHA game. Let $T \subseteq N, i \in N \backslash T$. Then, the marginal contribution of node $i$ to $T$ is

$$
v(T \cup\{i\})-v(T)=p_{i} \sum_{\substack{D \subseteq T \\|D| \geq 1}} \prod_{j \in D} p_{j} \prod_{j \in N \backslash(D \cup\{i\})}\left(1-p_{j}\right) .
$$

Proof. By Proposition 3, we find

$$
\begin{aligned}
v(T \cup\{i\})-v(T) & =\sum_{\substack{D \subseteq T \cup\{i\} \\
|D| \geq 2}} \prod_{j \in D} p_{j} \prod_{j \in N \backslash D}\left(1-p_{j}\right)-\sum_{\substack{D \subseteq T \\
|D| \geq 2}} \prod_{j \in D} p_{j} \prod_{j \in N \backslash D}\left(1-p_{j}\right) \\
& =\sum_{\substack{D \subseteq T \cup\{i\} \\
|D| \geq 2 \\
i \in \bar{D}}} \prod_{j \in D} p_{j} \prod_{j \in N \backslash D}\left(1-p_{j}\right) \\
& =p_{i} \sum_{\substack{D \subseteq T \\
|D| \geq 1}} \prod_{j \in D} p_{j} \prod_{j \in N \backslash(D \cup\{i\})}\left(1-p_{j}\right) .
\end{aligned}
$$

Observe that (14) can be identified as the probability that node $i$ is active, no nodes in $N \backslash(T \cup\{i\})$ are active, and at least one node in $T$ is active. Indeed, intuitively this is the only way in which coalition $T \cup\{i\}$ can improve upon $T$.

Theorem 1. Let $(N, p)$ be an ALOHA situation and let $(N, v)$ be the corresponding ALOHA game. Then $(N, v)$ is convex.

Proof. Let $i \in N$ and $S \subseteq T \subseteq N \backslash\{i\}$. By Lemma 1 we have

$$
\begin{aligned}
v(T \cup\{i\})-v(T) & =p_{i} \sum_{\substack{D \subseteq T \\
|D| \geq 1}} \prod_{j \in D} p_{j} \prod_{j \in N \backslash(D \cup\{i\})}\left(1-p_{j}\right) \\
& \geq p_{i} \sum_{\substack{D \subseteq S \\
|D| \geq 1}} \prod_{j \in D} p_{j} \prod_{j \in N \backslash(D \cup\{i\})}\left(1-p_{j}\right)=v(S \cup\{i\})-v(S) .
\end{aligned}
$$

Hence, $(N, v)$ is convex.

\section{Solution concepts}

In this section we use the cooperative ALOHA game introduced in Section 4 to study the allocation of the throughput gains. We analyze three solution concepts of the ALOHA game: the core, the Shapley value, and the compromise value.

From Theorem 1 it follows that the core can be written as the convex hull of the marginal vectors. By exploiting the specific structure of an ALOHA game we can derive the following expression for these marginal vectors. 


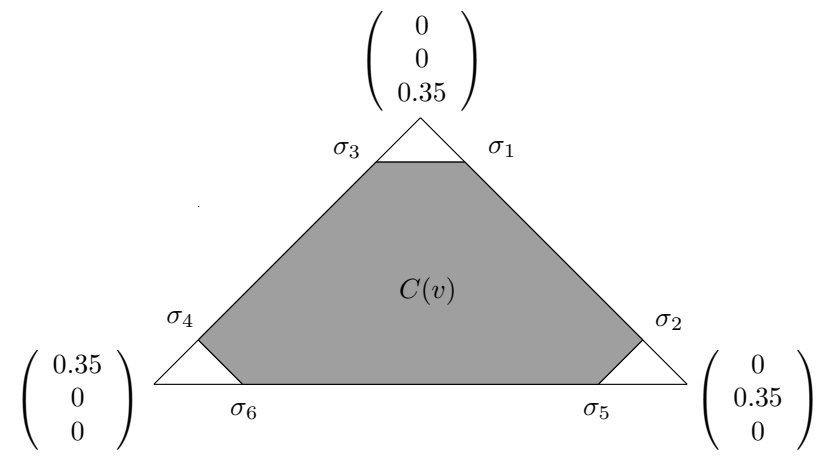

Figure 1: The core of the game in Example 3.

Lemma 2. Let $(N, p)$ be an ALOHA situation and let $(N, v)$ be the corresponding ALOHA game. Let $\sigma \in \Pi(N)$ be a permutation, then the marginal vector $m^{\sigma}(v)$ is given by

$$
m_{i}^{\sigma}(v)=p_{i} \sum_{\substack{D \subseteq \Omega^{\sigma}(\sigma(i)-1) \\|D| \geq 1}} \prod_{j \in D} p_{j} \prod_{j \in N \backslash(D \cup\{i\})}\left(1-p_{j}\right), \quad i \in N .
$$

Proof. This readily follows from applying Lemma 1 to the definition of the marginal vector (1), with $T=\Omega^{\sigma}(\sigma(i)-1)$.

To illustrate the connection between the core and the marginal vectors, we revisit the ALOHA game of Example 2.

Example 3. The ALOHA game of Example 2 is given by

\begin{tabular}{|c||c|c|c|c|c|c|c|}
\hline$S$ & 1 & 2 & 3 & 12 & 23 & 13 & 123 \\
\hline$v(S)$ & 0 & 0 & 0 & 0.06 & 0.09 & 0.14 & 0.35 \\
\hline
\end{tabular}

and the six marginal vectors $m^{\sigma_{i}}(v)$ are

\begin{tabular}{|l|l|}
\hline$\sigma_{1}=(1,2,3)$ & $m^{\sigma_{1}}(v)=(0,0.06,0.29)$ \\
\hline$\sigma_{2}=(1,3,2)$ & $m^{\sigma_{2}}(v)=(0,0.21,0.14)$ \\
\hline$\sigma_{3}=(2,1,3)$ & $m^{\sigma_{3}}(v)=(0.06,0,0.29)$ \\
\hline$\sigma_{4}=(2,3,1)$ & $m^{\sigma_{4}}(v)=(0.26,0,0.09)$ \\
\hline$\sigma_{5}=(3,1,2)$ & $m^{\sigma_{5}}(v)=(0.14,0.21,0)$ \\
\hline$\sigma_{6}=(3,2,1)$ & $m^{\sigma_{6}}(v)=(0.26,0.09,0)$ \\
\hline
\end{tabular}

Here, $m^{\sigma_{3}}(v)$ is for example calculated as

$$
\left(\begin{array}{l}
m_{\sigma(1)}^{\sigma_{3}}(v) \\
m_{\sigma(2)}^{\sigma_{3}}(v) \\
m_{\sigma(3)}^{\sigma_{3}}(v)
\end{array}\right)=\left(\begin{array}{l}
m_{2}^{\sigma_{3}}(v) \\
m_{1}^{\sigma_{3}}(v) \\
m_{3}^{\sigma_{3}}(v)
\end{array}\right)=\left(\begin{array}{l}
v(2) \\
v(21)-v(2) \\
v(213)-v(21)
\end{array}\right)=\left(\begin{array}{l}
0 \\
0.06 \\
0.29
\end{array}\right) .
$$

Hence,

$C(v)=\operatorname{conv}\{(0,0.06,0.29),(0,0.21,0.14),(0.06,0,0.29),(0.26,0,0.09),(0.14,0.21,0),(0.26,0.09,0)\}$, which is displayed in Figure 1.

Next, we analyze the relation between the core and the allocations of the WGS rules. The following lemma states that any allocation prescribed by a WGS rule is an element of the core.

Lemma 3. Let $(N, p)$ be an ALOHA situation and let $(N, v)$ be the corresponding ALOHA game. Then $W G S(p) \subseteq C(v)$. 
Proof. Let $\lambda \in \Lambda$. By Proposition 2 we know that $W G S^{\lambda}(p)$ is efficient, and it remains to show that $\sum_{i \in S} W G S_{i}^{\lambda}(p) \geq v(S)$ for any $S \subseteq N$.

Let $S \subseteq N$. Then,

$$
\begin{aligned}
\sum_{i \in S} W G S_{i}^{\lambda}(p) & =\sum_{i \in S} \sum_{\substack{D \subseteq N \\
i \in D}} \lambda_{i}^{D} \prod_{j \in D} p_{j} \prod_{j \in N \backslash D}\left(1-p_{j}\right) \geq \sum_{i \in S} \sum_{\substack{D \subseteq S \\
i \in D \\
|D| \geq 2}} \lambda_{i}^{D} \prod_{j \in D} p_{j} \prod_{j \in N \backslash D}\left(1-p_{j}\right) \\
& =\sum_{\substack{D \subseteq S \\
|D| \geq 2}} \sum_{i \in D} \lambda_{i}^{D} \prod_{j \in D} p_{j} \prod_{j \in N \backslash D}\left(1-p_{j}\right)=\sum_{\substack{D \subseteq S \\
|D| \geq 2}} \prod_{j \in D} p_{j} \prod_{j \in N \backslash D}\left(1-p_{j}\right)=v(S) .
\end{aligned}
$$

Hence, $W G S^{\lambda}(p) \in C(v)$ for all $\lambda \in \Lambda$.

It turns out that the converse is also true.

Lemma 4. Let $(N, p)$ be an ALOHA situation and let $(N, v)$ be the corresponding ALOHA game. Then, $C(v) \subseteq W G S(p)$.

Proof. Let $x \in C(v)$. By (2), we can write

$$
x=\sum_{\sigma \in \Pi(N)} \mu^{\sigma} m^{\sigma}(v),
$$

for some $\mu=\left(\mu^{\sigma}\right)_{\sigma \in \Pi(N)}$ such that $\sum_{\sigma} \mu^{\sigma}=1$ and $\mu^{\sigma} \geq 0$ for all $\sigma \in \Pi(N)$. Since $W G S(p)$ is convex for all $p$, it suffices to show for each $\sigma \in \Pi(N)$ that there exists $\lambda \in \Lambda$ such that $m^{\sigma}(v)=W G S^{\lambda}(p)$.

Let $\sigma \in \Pi(N)$. Using Lemma 2, we have

$$
\begin{aligned}
m_{i}^{\sigma}(v) & =p_{i} \sum_{\substack{D \subseteq \Omega^{\sigma}(\sigma(i)-1) \\
|D| \geq 1}} \prod_{j \in D} p_{j} \prod_{j \in N \backslash(D \cup\{i\})}\left(1-p_{j}\right)=\sum_{\substack{D \subseteq \Omega^{\sigma}(\sigma(i)) \\
i \in D \\
|D| \geq 2}} \prod_{j \in D} p_{j} \prod_{j \in N \backslash D}\left(1-p_{j}\right) \\
& =\sum_{\substack{D \subseteq N \\
i \in D \\
|D| \geq 2}} I_{\left\{D \subseteq \Omega^{\sigma}(\sigma(i))\right\}} \prod_{j \in D} p_{j} \prod_{j \in N \backslash D}\left(1-p_{j}\right)=W G S_{i}^{\lambda}(p),
\end{aligned}
$$

where $\lambda=\left\{\lambda^{D}\right\}_{D \subseteq N}$ is defined as

$$
\lambda_{j}^{D}= \begin{cases}1 & \text { if } j \in D \text { and } D \subseteq \Omega^{\sigma}(\sigma(j)) \\ 0 & \text { otherwise }\end{cases}
$$

for all $D \subseteq N$.

As a consequence of Lemma 3 and Lemma 4, the core and the set of all WGS rules coincide.

Theorem 2. Let $(N, p)$ be an ALOHA situation and let $(N, v)$ be the corresponding ALOHA game. Then,

$$
W G S(p)=C(v) .
$$

For convex games, a specific element of the core is the Shapley value. As ALOHA games are convex, and the set of WGS rules spans the entire core, there exist specific elements of $\Lambda$ that generate the Shapley value.

Theorem 3. Let $(N, p)$ be an ALOHA situation and let $(N, v)$ be the corresponding ALOHA game. Choose $\lambda=\left\{\lambda^{D}\right\}_{D \subseteq N} \in \Lambda$ as

$$
\lambda_{i}^{D}= \begin{cases}\frac{1}{|D|} & \text { if } i \in D \\ 0 & \text { if } i \in N \backslash D,\end{cases}
$$

for all $D \subseteq N$. Then,

$$
\Phi(v)=W G S^{\lambda}(p)
$$


Proof. Recall from (3) that for $i \in N$ we have

$$
\Phi_{i}(v)=\frac{1}{|N| !} \sum_{\sigma \in \Pi(N)} m_{i}^{\sigma}(v) .
$$

By Lemma 4 we have that

$$
\Phi_{i}(v)=\frac{1}{|N| !} \sum_{\sigma \in \Pi(N)} \sum_{\substack{D \subseteq N \\ i \in D \\|D| \geq 2}} \mathrm{I}_{\left\{D \subseteq \Omega^{\sigma}(\sigma(i))\right\}} \prod_{j \in D} p_{j} \prod_{j \in N \backslash D}\left(1-p_{j}\right) .
$$

By counting the number of permutations for which all elements of the set $D$ are positioned before node $i$, we find

$$
\frac{1}{|N| !} \sum_{\sigma \in \Pi(N)} \mathrm{I}_{\left\{D \subseteq \Omega^{\sigma}(\sigma(i))\right\}}=\frac{1}{|N| !} \sum_{k=|D|}^{|N|}\left(\begin{array}{c}
k-1 \\
|D|-1
\end{array}\right)(|N|-|D|) !(|D|-1) !=\frac{1}{|D|},
$$

where the second equality follows from the combinatorial identity $\sum_{j=k}^{n}\left(\begin{array}{l}j \\ k\end{array}\right)=\left(\begin{array}{l}n+1 \\ k+1\end{array}\right)$. Substituting (16) into (15),

$$
\Phi_{i}(v)=\sum_{\substack{D \subseteq N \\ i \in D \\|D| \geq 2}} \frac{1}{|D|} \prod_{j \in D} p_{j} \prod_{j \in N \backslash D}\left(1-p_{j}\right)=W G S_{i}^{\lambda}(p) .
$$

From Theorems 2 and 3, and the fact that WGS rules depend solely on the transmission probabilities of the nodes, it follows that both the core and the Shapley value can be obtained without any explicit knowledge of the ALOHA game. Another example of a solution concept that can be obtained independently from the game, is given in Hamers et al. [6]. In this paper, the authors consider a one-machine sequencing situation in which each agent has one job that has to be processed. They propose a solution concept that depends only on the processing times of the jobs and the weights in the cost function. This rule constitutes only a subset of the core, and explicit knowledge of the game is required to obtain the entire core.

The next theorem provides an explicit expression for the compromise value of ALOHA games. This value allocates to each node $k$ a fraction $w_{k}$ of $v(N)$. The numerator of $w_{k}$ can be interpreted as the probability that node $k$ is involved in a collision in an arbitrary time slot. So $w_{k}$ is a measure of the loss in throughput incurred from activity of node $k$. That is, it gives an indication of the gain obtained by adding node $k$ to the coalition.

Theorem 4. Let $(N, p)$ be an ALOHA situation and let $(N, v)$ be the corresponding ALOHA game. Then, $\tau_{k}(v)=w_{k} v(N)$, where

$$
w_{k}=\frac{p_{k}\left(1-\prod_{j \in N \backslash\{k\}}\left(1-p_{j}\right)\right)}{\sum_{i \in N} p_{i}\left(1-\prod_{j \in N \backslash\{i\}}\left(1-p_{j}\right)\right)}
$$

for all $k \in N$.

Proof. By Theorem 1, we know that $(N, v)$ is convex. Since $v(\{k\})=0$ for all $k \in N$, the compromise value is of the form

$$
\tau(v)=\beta M(v),
$$

where $\beta$ is a normalizing constant. By applying the definition of $M_{k}(v)$ from (4),

$$
\begin{aligned}
M_{k}(v) & =p_{k} \sum_{\substack{D \subseteq N \backslash\{k\} \\
|D| \geq 1}} \prod_{i \in D} p_{i} \prod_{i \in N \backslash(D \cup\{k\})}\left(1-p_{i}\right) \\
& =p_{k}\left(1-\prod_{j \in N \backslash\{k\}}\left(1-p_{j}\right)\right),
\end{aligned}
$$

where the second equality follows from Lemma 1 . The result then follows by substituting (18) into (17), and computing the normalization constant. 


\section{Sensitivity analysis}

In the previous section, we presented a number of solution concepts that designate the throughput gains of cooperation among the nodes. We are interested how sensitive these solution concepts are to changes in the ALOHA situation. Specifically, we investigate how a WGS allocation responds to changes in the transmission probabilities, and how the Shapley value is affected when the number of nodes changes. Thirdly, we consider the case where the transmission probabilities of all nodes are equal. In this case, the expression for the Shapley value simplifies and allows for a more detailed sensitivity analysis.

Let $\lambda \in \Lambda$ and consider the corresponding WGS rule. It is easy to see that the gains allocated to node $i$ are increasing in $p_{i}$. That is,

$$
\frac{\partial}{\partial p_{i}} W G S_{i}^{\lambda}(p)=\sum_{\substack{D \subseteq N \\ i \in D \\|D| \geq 2}} \lambda_{i}^{D} \prod_{j \in D \backslash\{i\}} p_{j} \prod_{j \in N \backslash D}\left(1-p_{j}\right)>0 .
$$

Note that since the Shapley value can be characterized as a specific WGS rule, also the Shapley value for player $i$ of the corresponding ALOHA game will be increasing in $p_{i}$.

How the WGS allocation to node $i$ is affected by changes to the transmission probability of another node $k$ changes is less straightforward. In fact, depending on the choice of $\lambda$, we see that $\frac{\partial}{\partial p_{k}} W G S_{i}^{\lambda}(p)$ may be either negative or positive. For example, let $i, k \in\{1,2, \ldots,|N|\}, i \neq k$, and choose $\lambda \in \Lambda$ such that

$$
\lambda_{i}^{D}= \begin{cases}1 & i \in D \text { and } k \in D \\ 0 & \text { otherwise }\end{cases}
$$

for all $D \subseteq N$. Then,

$$
W G S_{i}^{\lambda}(p)=\sum_{\substack{D \subseteq N \\ i, k \in D \\|D| \geq 2}} \prod_{j \in D} p_{j} \prod_{j \in N \backslash D}\left(1-p_{j}\right)
$$

and

$$
\frac{\partial}{\partial p_{k}} W G S_{i}^{\lambda}(p)=\sum_{\substack{D \subseteq N \backslash\{k\} \\ i \in D \\|D| \geq 2}} \prod_{j \in D} p_{j} \prod_{j \in N \backslash D}\left(1-p_{j}\right)>0 .
$$

Conversely, if we choose $\lambda \in \Lambda$ such that

$$
\lambda_{i}^{D}= \begin{cases}1 & i \in D, \quad k \notin D \\ 0 & \text { otherwise }\end{cases}
$$

for all $D \subseteq N$, we find

$$
\frac{\partial}{\partial p_{k}} W G S_{i}^{\lambda}(p)=-\sum_{\substack{D \subseteq N \backslash\{k\} \\ i \in D \\|D| \geq 2}} \prod_{j \in D} p_{j} \prod_{j \in N \backslash(D \cup\{k\})}\left(1-p_{j}\right)<0 .
$$

Next, we investigate the impact on the Shapley value of expanding the network of ALOHA situation $(N, p)$ by a single node. To this end we introduce a second ALOHA situation $(\hat{N}, \hat{p})$ with $\hat{N}=N \cup\{t\}$ and $\hat{p}=\left(p_{1}, \ldots, p_{n}, p_{t}\right)$. We can express the Shapley value of the extended ALOHA situation in the Shapley value of the original game as follows.

Proposition 4. Let $(N, p)$ and $(\hat{N}, \hat{p})$ be the two ALOHA situations described above and let $(N, v)$ and $(\hat{N}, \hat{v})$ denote the respective corresponding ALOHA games. Then

$$
\Phi_{i}(\hat{v})=p_{t} \sum_{\substack{D \subseteq N \\ i \in D \\|D| \geq 1}} \frac{1}{|D|+1} \prod_{j \in D} p_{j} \prod_{j \in N \backslash D}\left(1-p_{j}\right)+\left(1-p_{t}\right) \Phi_{i}(v), \quad i \in N .
$$


Proof. Let $i \in N$. By Theorem 3, we have

$$
\begin{aligned}
\Phi_{i}(\hat{v}) & =\sum_{\substack{D \subseteq \hat{N} \\
i \in D \\
|D| \geq 2}} \frac{1}{|D|} \prod_{j \in D} p_{j} \prod_{j \in \hat{N} \backslash D}\left(1-p_{j}\right) \\
& =\sum_{\substack{D \subseteq \hat{N} \\
i \in D, t \in D \\
|D| \geq 2}} \frac{1}{|D|} \prod_{j \in D} p_{j} \prod_{j \in \hat{N} \backslash D}\left(1-p_{j}\right)+\sum_{\substack{D \subseteq \hat{N} \\
i \in D, t \in N \backslash D \\
|D| \geq 2}} \frac{1}{|D|} \prod_{j \in D} p_{j} \prod_{j \in \hat{N} \backslash D}\left(1-p_{j}\right) \\
& =p_{t} \sum_{\substack{D \subseteq N \\
i \in D \\
|D| \geq 1}} \frac{1}{|D|+1} \prod_{j \in D} p_{j} \prod_{j \in N \backslash D}\left(1-p_{j}\right)+\left(1-p_{t}\right) \sum_{\substack{D \subseteq N \\
i \in D \\
|D| \geq 2}} \frac{1}{|D|} \prod_{j \in D} p_{j} \prod_{j \in N \backslash D}\left(1-p_{j}\right)
\end{aligned}
$$

By the definition of $\Phi_{i}(v)$, we get (19).

We can interpret (19) as the Shapley value conditioned on the activity of node $t$. When node $t$ is inactive (with probability $1-p_{t}$ ) the Shapley value reduces to that of the system without node $t$ present.

Now consider the case where all nodes have equal transmission probabilities, i.e., $p_{i}=\rho$, $i=1,2, \ldots,|N|$. In this case, the sensitivity analysis simplifies considerably. In particular, the Shapley value may be written in a more explicit form.

Proposition 5. Let $(N, p)$ be an ALOHA situation, with $p_{i}=\rho$ for all $i=1,2, \ldots,|N|$, and let $(N, v)$ be the corresponding ALOHA game. Then,

$$
\Phi_{i}(v)=\frac{1}{|N|}\left(1-(1-\rho)^{|N|}-|N| \rho(1-\rho)^{|N|-1}\right), \quad i \in N .
$$

Proof. By Theorem 3 and summing over all possible sizes of subsets $D \subseteq N$ with node $i$, we find

$$
\begin{aligned}
\Phi_{i}(v) & =\sum_{\substack{D \subseteq N \\
i \in D \\
|D| \geq 2}} \frac{1}{|D|} \rho^{|D|}(1-\rho)^{|N|-|D|}=\sum_{m=2}^{|N|} \frac{1}{m}\left(\begin{array}{c}
|N|-1 \\
m-1
\end{array}\right) \rho^{m}(1-\rho)^{|N|-m} \\
& =\frac{1}{|N|} \sum_{m=2}^{|N|}\left(\begin{array}{c}
|N| \\
m
\end{array}\right) \rho^{m}(1-\rho)^{|N|-m}, \quad i \in N .
\end{aligned}
$$

The result is then obtained by applying the binomial theorem to (21).

The expression between brackets in (20) represents the fraction of slots in which at least two nodes attempt to transmit a packet. This corresponds to throughput gains,as such attempts would result in a collision if not for cooperation. The prefactor $1 /|N|$ is explained by the fact that all nodes have equal transmission probability, so the gains are spread evenly among all nodes.

Taking the derivative of $(20)$ with respect to $\rho$, we see that the Shapley value is increasing in the transmission probability,

$$
\frac{\partial}{\partial \rho} \Phi_{i}(v)=(|N|-1) \rho(1-\rho)^{|N|-2}>0, \quad i \in N .
$$

Indeed, increasing all transmission probabilities leads to more collisions and induces higher gains from cooperation. As these gains are distributed uniformly among all nodes, the allocation by the Shapley value is increasing in $\rho$.

Finally, we consider the impact on the Shapley value of expanding the network by a single node, given that all nodes have equal transmission probability. In this case, (19) simplifies to

$$
\Phi_{i}(\hat{v})=\frac{1}{|N|+1}\left(\rho-\rho(1-\rho)^{|N|}\right)+\frac{|N|}{|N|+1}(1-\rho) \Phi_{i}(v), \quad i \in N .
$$


It is not immediate from (22) how adding a node affects the allocation done by the Shapley value to a specific node. Indeed, depending on $|N|$ and $\rho$ the Shapley value may either increase or decrease.

Let $\Phi(n, \rho)$ denote the Shapley value of a game with $n=|N|$ nodes and equal transmission probability $\rho$ and consider $n^{*}(\rho)=\max _{n} \Phi(n, \rho)$, the number of nodes that maximizes the Shapley value given $\rho$. It is readily seen that for $\rho$ sufficiently large, $n^{*}(\rho)=2$, and that $n^{*}(\rho) \rightarrow \infty$ as $\rho \downarrow 0$. Adding a node to the network increases the gains from cooperation as the number of collisions are decreased, but also requires these gains to be shared among more nodes. Hence, for $\rho$ small enough, the increase in gains outweighs the decrease in the Shapley value due to the addition of an extra node, and for $\rho$ close to 1, the probability of a collision is already large, and the increase in gains does not offset the additional node.

\section{References}

[1] Abramson, N. (1970). The ALOHA System - Another Alternative for Computer Communications. AFIPS Conference Proceedings. Vol. 36, pp. 295-298.

[2] Aram, A., Singh, C., Sarkar, S. and Kumar, A. (2009). Cooperative Profit Sharing in Coalition Based Resource Allocation in Wireless Networks. In proceedings of INFOCOM 2009, pp. 21232131.

[3] Calleja, P., Borm, P., Hendrickx, R. (2005). Multi-issue allocation situations. European Journal of Operational Research. Vol. 164, pp. 730-747.

[4] Driessen, T., Tijs, S.H. (1985). The $\tau$-value, the core and semiconvex games. International Journal of Game Theory. Vol. 14, pp. 229-247.

[5] Gillies, D.B. (1953). Some theorems on $n$-person games. Dissertation, Department of Mathematics, Princeton University.

[6] Hamers, H., Suijs, J., Tijs, S.H. and Borm, P. (1996). The split core for sequencing games Games and Economic Behaviour. Vol. 15, pp. 165-176.

[7] Ichiishi, T. (1981). Super-Modularity: Applications to Convex Games and the Greedy Algorithm for LP. Journal of Economic Theory, Vol. 25, pp. 283286.

[8] La, R., Anantharam, V. (2004). A Game-theoretic Look at the Gaussian Multiaccess Channel. Advances in Network Information Theory. Vol. 66, pp. 87-105.

[9] Mathur, S. and Sankaranarayanan, L. and Mandayam, N. (2006). Coalitional Games in Gaussian Interference Channels. In proceedings of IEEE ISIT 2006, pp. 2210-2214.

[10] Roberts, L. (1975). ALOHA Packet System with and without Slots and Capture. Computer Communication Review. Vol 5, pp. 28-42.

[11] Rom, R. and Sidi, M. (1990). Multiple Access Protocols. Springer-Verlag, New York.

[12] Shapley, S. (1953). A value for $n$-person games. Annals of Mathematics Study. Vol. 28, pp. 307-317.

[13] Shapley, S. (1971). Cores of Convex Games. International Journal of Game Theory. Vol. 1, pp. 11-26.

[14] Tijs, S.H. (1981). Bounds for the core and the $\tau$-value. In: Game Theory and Mathematical Economics, O. Moeschlin and D. Pallaschke (eds.), North-Holland, Amsterdam, pp. 123-132. 